\title{
Improved near-well approximation for prediction of the gas/oil production ratio from oil-rim reservoirs
}

\author{
Svenn Anton Halvorsen • Are Mjaavatten • \\ Robert Aasheim
}

Received: 26 January 2011 / Accepted: 26 July 2011 / Published online: 6 September 2011

(C) The Author(s) 2011. This article is published with open access at Springerlink.com

\begin{abstract}
A model with short computational time has previously been developed to predict the ratedependent gas/oil ratio (GOR) from a horizontal well. The oil flow towards the wellbore is based on a onedimensional model by Konieczek. The model performs remarkably well for medium-time production optimization (weeks, months), while the predictions during the first days after a large change in the production can be poor. An improved one-dimensional model for the flow towards the wellbore is proposed, where the oil flow is treated as a superposition of three terms:
\end{abstract}

1) Radial flow towards the wellbore and towards a mirror well.

2) Flow to correct for modified boundary conditions due to the radial flows.

3) Flow due to height variations of the gas/oil contact (GOC).

The new model takes care of the current short term and near-well deficiencies: Effect of 2D flow close to the wellbore, gas breakthrough due to viscous gas fingering, and horizontal/vertical anisotropy. Based on analysis and preliminary testing the new model should have equally good medium and long term capabilities

S. A. Halvorsen $(\bowtie)$

Teknova AS, Kristiansand, Norway

e-mail: sah@teknova.no

\section{A. Mjaavatten}

Yara International ASA, Porsgrunn, Norway

R. Aasheim

Statoil ASA, Research Centre Porsgrunn,

Porsgrunn, Norway and considerably improved short term and near-well behaviour, compared to the present implementation.

Keywords Oil-rim reservoir • Horizontal well • Improved near-well model • Gas coning • Gas/oil production ratio - GOR • Gas breakthrough • GORM • One-dimensional model •

Production optimization - Gravitational drainage • Dupuit-Forchheimer - Porous media equation

\section{Nomenclature}

$\alpha \quad$ Parameter in the Dupuit-Forchheimer (porous media) equation, $\mathrm{m} / \mathrm{s}$

$\beta \quad$ Non-dimensional model parameter to be adapted to the production data, used to define the distribution of the weighted production rate along the wellbore

$\delta \quad$ Non-dimensional parameter applied in GORM to define the oil reduction factor, $\kappa$

$\delta_{\mathrm{a}} \quad$ Aspect ratio, adjusted for the effect of different permeabilities, $\delta_{\mathrm{a}}=\sqrt{\frac{k_{\mathrm{h}}}{k_{\mathrm{v}}}} \frac{h_{0}}{W}$

$\Delta \rho \quad$ Density difference between oil and gas in the reservoir, $\mathrm{kg} / \mathrm{m}^{3}, \Delta \rho=\rho_{\mathrm{o}}-\rho_{\mathrm{g}}$

$\gamma \quad$ Non-dimensional model parameter for defining the weighted (local) production rate, $\gamma \mathrm{m}^{2} / \mathrm{s}$ free gas corresponds to $1 \mathrm{~m}^{2} / \mathrm{s}$ local oil production

$\varphi \quad$ Effective porosity, $\mathrm{m}^{3} / \mathrm{m}^{3}$

$\kappa \quad$ Non-dimensional oil reduction factor. When free gas is produced, the local oil production is reduced by the factor $\kappa$ 
$\mu \quad$ Oil viscosity, $\mathrm{Pa} \mathrm{s}$

$\rho_{\mathrm{o}} \quad$ Density of oil in the reservoir, $\mathrm{kg} / \mathrm{m}^{3}$

$\rho_{\mathrm{g}} \quad$ Density of gas in the reservoir, $\mathrm{kg} / \mathrm{m}^{3}$

$\tau_{\mathrm{h}}$

$\tau_{\mathrm{v}}$

$B_{\mathrm{g}}$

$B_{\text {o }}$

$c$

$c_{\mathrm{h}}$

$c_{\mathrm{V}}$

$d_{\mathrm{W}}$

$g$

GOC

$h$

$h_{0}$

$h_{1}$

$h_{2}$

$k$

$k_{\mathrm{h}}$

$k_{\mathrm{V}}$

$K(y)$

$K_{\text {I }}$

$K_{\text {red }}$

L

p

$\widetilde{p}$

$p_{\text {fix }}$

$p_{\text {gravity }}$

$p_{\mathrm{h}}$

$p_{\text {ref }}$

$p_{\text {sink }}$

$p_{\text {tot }}$

$p_{0}$

$q_{\mathrm{a}}$

Time constant for horizontal flow, $\mathrm{s}$

Time constant for vertical flow, $\mathrm{s}$

Gas formation factor, $\mathrm{m}^{3} / \mathrm{Sm}^{3}$. One standard $\mathrm{m}^{3}$ gas has a volume of $B_{\mathrm{g}} \mathrm{m}^{3}$ in the reservoir

Oil formation factor, $\mathrm{m}^{3} / \mathrm{Sm}^{3}$. One standard $\mathrm{m}^{3}$ oil has a volume of $B_{0} \mathrm{~m}^{3}$ in the reservoir Isotropic diffusion coefficient for oil flow, $\mathrm{m}^{2} /$ Pa s, $c=\frac{k}{\mu}$

Diffusion coefficient in the horizontal direction, $\mathrm{m}^{2} / \mathrm{Pa} \mathrm{s}, c_{\mathrm{h}}=\frac{k_{\mathrm{h}}}{\mu}$

Diffusion coefficient in the vertical direction, $\mathrm{m}^{2} / \mathrm{Pa} \mathrm{s}, c_{\mathrm{v}}=\frac{k_{\mathrm{v}}}{\mu}$

Diameter of the wellbore, $\mathrm{m}$

$9.869 \mathrm{~m} / \mathrm{s}^{2}$, gravity constant

Gas oil contact, i.e. the interface between oil and gas

Local height of the oil layer, $\mathrm{m}, h=h(x, y, t)$

Initial height of the oil reservoir, $m$

Local height of the first cell in GORM, m, $h_{1}(y, t) \approx h(0, y, t)$

Local height of the second cell in GORM, $m$

Isotropic permeability, $\mathrm{m}^{2}$

Horizontal permeability, $\mathrm{m}^{2}$

Vertical permeability, $\mathrm{m}^{2}$

Non-dimensional function for the distribution of weighted production along the wellbore

(Weighted) production integral, integral of $K(y)$ along the wellbore, $\mathrm{m}$

oil reduction integral defined by $K_{\text {red }}=$ $\int_{0}^{L} \kappa(y, t) K(y) d y$

Length of the wellbore, $\mathrm{m}$

Deviatoric pressure, i.e. pressure causing flow, $\mathrm{Pa}$

Non-dimensional deviatoric pressure

Deviatoric pressure to counteract (fix) the

boundary conditions due to $p_{\text {sink }}, \mathrm{Pa}$

Part of deviatoric pressure due to gravity, caused by variations of GOC, $\mathrm{Pa}$

Hydrostatic pressure in the reservoir, $\mathrm{Pa}$

Reference pressure, i.e. hydrostatic (gas)

pressure at the reference level, $\mathrm{Pa}$

Deviatoric pressure due to two sinks, $\mathrm{Pa}$

Total pressure in the reservoir, $\mathrm{Pa}$

Typical pressure difference, $\mathrm{Pa}, p_{0}=\Delta \rho \mathrm{gh}_{0}$

Total weighted production rate at the reservoir, $\mathrm{m}^{3} / \mathrm{s}$ $q_{\mathrm{g}}$

$q_{\mathrm{o}}$

$\bar{q}_{\mathrm{a}}$

$\bar{q}_{\mathrm{g}}$

$\bar{q}_{\mathrm{o}}$

$Q_{\mathrm{g}}$

$R_{\mathrm{S}}$

$t$

$u(t)$

$v_{\mathrm{c}}$

$v_{\mathrm{s}}$

$v_{\mathrm{X}}$

$v_{\mathrm{y}}$

$v_{\mathrm{Z}}$

$v_{\text {fix_x }}$

$V_{\text {gravity_x }}$

$V_{\text {sink_x }}$

$V_{\mathrm{x}}$

$V_{\mathrm{y}}$

W

$x$

$\tilde{x}$

$y$

$z$

$\widetilde{z}$

$z_{\text {ref }}$

$z_{\mathrm{W}}$
Total production rate of free gas at the reservoir, $\mathrm{m}^{3} / \mathrm{s}$

Total oil production rate at the reservoir, $\mathrm{m}^{3} / \mathrm{s}$

Weighted (local) production rate per meter well length, $\mathrm{m}^{2} / \mathrm{s}, \bar{q}_{\mathrm{a}}(y, t) \stackrel{\text { def }}{=} \frac{\bar{q}_{\mathrm{g}}(y, t)}{\gamma}+$ $\bar{q}_{\mathrm{o}}(y, t)=u(t) K(y)$

Local production rate of free gas per meter well length, $\mathrm{m}^{2} / \mathrm{s}$

Local volumetric oil production rate per meter well length, $\mathrm{m}^{2} / \mathrm{s}$

Total gas production, $\mathrm{Sm}^{3} / \mathrm{s}$

Solution of gas in the oil, $\mathrm{Sm}^{3} / \mathrm{Sm}^{3}$. One standard $\mathrm{m}^{3}$ oil from the reservoir contains $R_{\mathrm{s}} \mathrm{Sm}^{3}$ dissolved gas

Time, $\mathrm{s}$

Time-dependent part of the weighted production rate per $\mathrm{m}$ well length, $\mathrm{m}^{2} / \mathrm{s}$, $\bar{q}_{\mathrm{a}}(y, t)=u(t) K(y)$

Stability limit for the (superficial) vertical downwards velocity, $\mathrm{m} / \mathrm{s}$

Tangential (superficial) oil velocity at GOC in the direction towards the wellbore, $\mathrm{m} / \mathrm{s}$

Superficial oil velocity component in the (horizontal) $x$-direction, $\mathrm{m} / \mathrm{s}$

Superficial oil velocity component in the (horizontal) $y$-direction, $\mathrm{m} / \mathrm{s}$

Superficial oil velocity component in the (vertical) $z$-direction, $\mathrm{m} / \mathrm{s}$

Volume flow of oil in the $x$-direction per $\mathrm{m}$ well due to $p_{\text {fix }}, \mathrm{m}^{2} / \mathrm{s}$

Volume flow of oil in the $x$-direction per $\mathrm{m}$ well due to gravity flow, $\mathrm{m}^{2} / \mathrm{s}$

Volume flow of oil in the $x$-direction per $\mathrm{m}$ well due to flow towards two sinks, $\mathrm{m}^{2} / \mathrm{s}$

Horizontal oil volume flow per $\mathrm{m}$ normal to the wellbore, $\mathrm{m}^{2} / \mathrm{s}=$ total volume flow of oil in the $x$-direction per $\mathrm{m}$ well, $\mathrm{m}^{2} / \mathrm{s}$

Horizontal oil volume flow per $\mathrm{m}$ parallel to the wellbore, $\mathrm{m}^{2} / \mathrm{s}$

Half width of the oil reservoir, $m$

$x$-coordinate (horizontal, perpendicular to the wellbore), $\mathrm{m}$

Non-dimensional $x$-coordinate

$y$-coordinate (horizontal, parallel to the wellbore), $\mathrm{m}$

$z$-coordinate (vertical), $\mathrm{m}$

Non-dimensional $z$-coordinate

Reference level, i.e. vertical level for the reference pressure, $\mathrm{m}$

Vertical well location (top of the wellbore), $\mathrm{m}$ 
$z_{\mathrm{wc}} \quad$ Vertical location of the well centre, $\mathrm{m}$

$\tilde{z}_{\mathrm{wc}} \quad$ Non-dimensional vertical location of the well centre

\section{Introduction}

In oil-rim fields, a thin oil layer lies between an aquifer and a gas cap. Oil can be produced from such fields by horizontal wells. The production will lower the local gas/oil contact (GOC) near the well in a process called gas coning. After some time, the GOC will come in contact with the wellbore close to the riser and the gas/oil ratio (GOR) from the well will then vary strongly with the production rate. The ability to predict this dependency is essential for production optimization.

A model for gas coning and prediction of GOR was introduced by Muskat in 1937 [5]. This early work considers the conditions for a vertical well. A model for horizontal wells in oil-rim fields is described by Konieczek [3]. His concepts have been extended by Statoil to include variations of gas and oil production along the wellbore [4].

Statoil's GOR model (called GORM) has been developed to predict the rate dependent GOR for periods of several months. The model describes the essential dynamic reservoir behaviour with a simplified interaction between the well and the reservoir. Historical oil and gas production rates are applied to fit three adjustable model parameters.

GORM has been extensively tested and adapted to historical production data, c.f. [4]. The model has short computational time and performs remarkably well for medium term production optimization (weeks, months). The predictions during the first days after a large change in the production can, however, be poor.

The model equations have been analysed and the behaviour close to the wellbore has been compared to more accurate finite element simulations [1]. The analysis revealed that the model had suitable medium term properties but that the short-term behaviour could be poor due to the chosen simplifications. Based on these results, an improved approximation was formulated.

\section{Current model}

\subsection{Basic assumptions}

The model was originally intended for short term production optimization (days, possibly a few weeks). It should be comparatively simple to enable short com- putational time but sufficiently sophisticated to include the essential reservoir behaviour. A few suitable parameters should further be available to tune the model to historical production data.

The model, GORM, applies the following assumptions [4]:

- The gas is assumed to behave like an inviscid fluid, compared to the viscous oil. Hence, the pressure along GOC is given by the hydrostatic gas pressure.

- The oil zone of the well drainage region initially has the form of a rectangular parallelepiped with an impermeable bottom and impermeable vertical boundaries.

- The vertical plane through the wellbore splits the reservoir into two symmetric parts.

- Vertical oil flow and flow parallel to the direction of the wellbore can be neglected and the local oil flow towards the wellbore can be described by the one-dimensional Dupuit-Forchheimer equation.

- The distribution of the total (weighted) production rate locally along the well is described explicitly by a linear function.

- Free gas is produced at locations where GOC is in contact with the wellbore. Here, the local oil production is reduced proportionally to the production of free gas determined by the amount of GOC in contact with the well.

Typical shape of GOC from a model simulation is shown in Fig. 1.

\subsection{Model equations}

In a Cartesian coordinate system, let the $x$-axis be normal and the $y$-axis parallel to the wellbore. The $z$-axis is pointing vertically upwards with $z=0$ at the top of the aquifer. The oil reservoir is initially (before any production) bounded by the planes $x=-W, x=$ $W, y=0, y=L, z=0$ and $z=h_{0}$, where $L$ is the length of the well, $W$ is the half width and $h_{0}$ the initial height of the oil layer. Above the oil, an infinite gas cap is assumed.

The hydrostatic pressure, $p_{\mathrm{h}}$, within the oil layer is given by:

$$
\begin{aligned}
p_{\mathrm{h}} & =p_{\text {ref }}+\rho_{\mathrm{g}} g\left(z_{\text {ref }}-h\right)+\rho_{\mathrm{o}} g(h-z) \\
& =\Delta \rho g h+p_{\text {ref }}-\rho_{\mathrm{o}} g z+\rho_{\mathrm{g}} g z_{\text {ref }}
\end{aligned}
$$

where $p_{\text {ref }}$ is the (hydrostatic) gas pressure at some reference level, $z_{\text {ref }}, \rho_{\mathrm{o}}$ and $\rho_{\mathrm{g}}$ the reservoir oil and gas densities, $g$ the gravity constant, $h(x, y, t)$ the local height of the oil layer, and $\Delta \rho=\rho_{\mathrm{o}}-\rho_{\mathrm{g}}$. 
Fig. 1 Schematic shape of GOC as predicted by the model. (Not to scale)

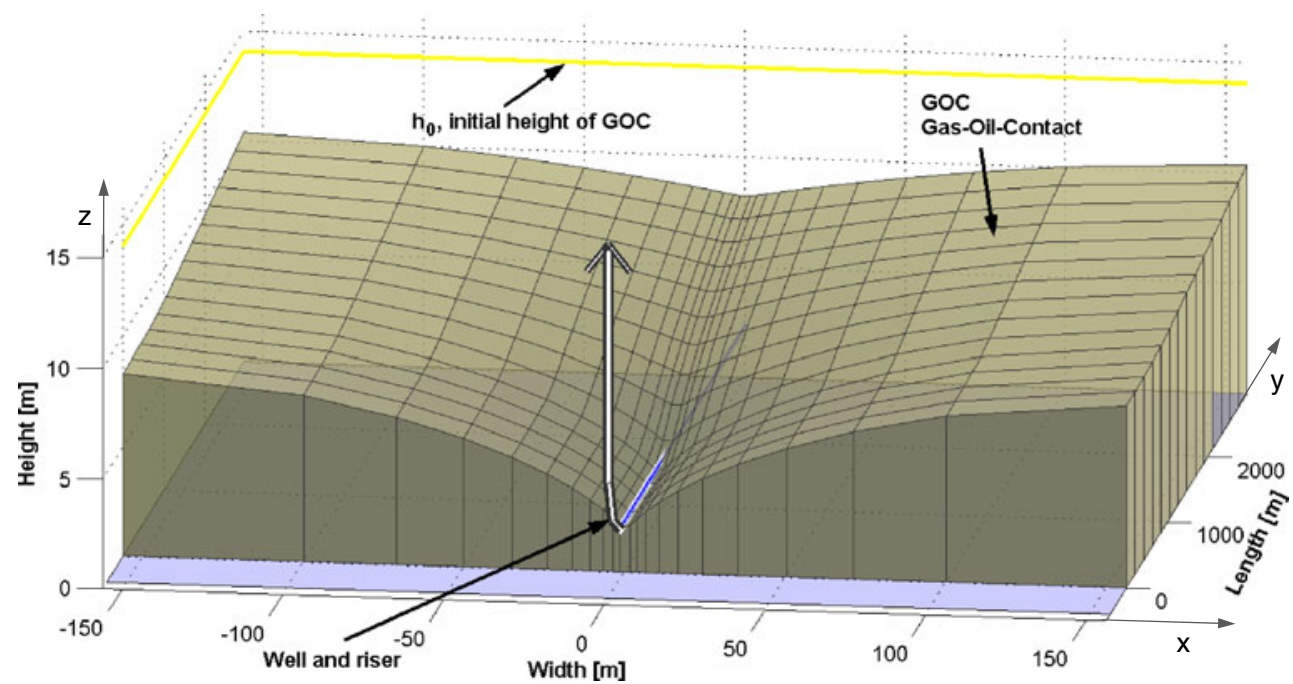

Let $p_{\text {tot }}$ be the total pressure and define the deviatoric pressure by:

$p=p_{\text {tot }}-\left(p_{\text {ref }}-\rho_{\mathrm{o}} g z+\rho_{\mathrm{g}} g z_{\text {ref }}\right)$

Oil flow is caused by gradients in the deviatoric pressure. Isotropic conditions are assumed in GORM [4]. Here, we will be slightly more general and assume constant, but possibly different permeabilities horizontally and vertically. The deviatoric pressure will then satisfy the equation:

$k_{\mathrm{h}} \frac{\partial^{2} p}{\partial x^{2}}+k_{\mathrm{h}} \frac{\partial^{2} p}{\partial y^{2}}+k_{\mathrm{v}} \frac{\partial^{2} p}{\partial z^{2}}=0$

and the superficial oil velocity components are given by:

$v_{\mathrm{x}}=-c_{\mathrm{h}} \frac{\partial p}{\partial x}, \quad v_{\mathrm{y}}=-c_{\mathrm{h}} \frac{\partial p}{\partial y}, \quad v_{\mathrm{z}}=-c_{\mathrm{v}} \frac{\partial p}{\partial z}$

where $k_{\mathrm{h}}$ and $k_{\mathrm{v}}$ are the horizontal and vertical permeabilities, $c_{\mathrm{h}}=\frac{k_{\mathrm{h}}}{\mu}, c_{\mathrm{v}}=\frac{k_{\mathrm{v}}}{\mu}$ where $\mu$ is the oil viscosity, and $v_{\mathrm{x}}, v_{\mathrm{y}}, v_{\mathrm{z}}$ are the superficial velocity components.

At GOC, the oil pressure is equal to the hydrostatic gas pressure. The total pressure is then equal to the hydrostatic pressure given by Eq. 1, and the deviatoric pressure is $p=\Delta \rho g h$ at GOC. The boundary conditions along the vertical and horizontal boundaries are zero normal pressure derivatives.

Mathematical analysis shows that the Dupuit assumption of vertical pressure equilibrium is a proper overall approximation [1]. The vertical pressure variation in the oil is then given by the hydrostatic pressure and the deviatoric pressure is:

$p(x, y, z, t)=\Delta \rho g h(x, y, t)$
According to Eqs. 4 and 5, the vertical oil velocity will be zero and the horizontal volume flows per $\mathrm{m}$ are given by:

$V_{\mathrm{x}}=h v_{\mathrm{x}}=-c_{\mathrm{h}} \Delta \rho g h \frac{\partial h}{\partial x}, \quad V_{\mathrm{y}}=h v_{\mathrm{y}}=-c_{\mathrm{h}} \Delta \rho g h \frac{\partial h}{\partial y}$

The material balance,

$\varphi \frac{\partial h}{\partial t}+\frac{\partial\left(h v_{\mathrm{x}}\right)}{\partial x}+\frac{\partial\left(h v_{\mathrm{y}}\right)}{\partial y}=0$,

then gives the two-dimensional Dupuit-Forchheimer (porous media) equation:

$\frac{\partial h}{\partial t}=\alpha\left(\frac{\partial}{\partial x}\left(h \frac{\partial h}{\partial x}\right)+\frac{\partial}{\partial y}\left(h \frac{\partial h}{\partial y}\right)\right)$

where $\alpha=\frac{c_{\mathrm{h}} \Delta \rho g}{\varphi}=\frac{k_{\mathrm{h}} \Delta \rho g}{\mu \varphi}$ and $\varphi$ is the effective porosity.

When the well length is more than three times the reservoir half width, the last term in Eq. 8 can be neglected [1], and the oil drainage can be approximated by the dominating term, as proposed by Konieczek [3]:

$\frac{\partial h}{\partial t}=\alpha \frac{\partial}{\partial x}\left(h \frac{\partial h}{\partial x}\right)$

As boundary conditions for Eq. 9, GORM applies the volumetric local oil production rate at $x=0$ :

$\frac{1}{2} \bar{q}_{\mathrm{o}}(y, t)=\left[-h v_{\mathrm{x}}\right]_{x=0}=\left[c_{\mathrm{h}} \Delta \rho g h \frac{\partial h}{\partial x}\right]_{x=0}$

and requires no-flow condition at $x=W$ :

$v_{\mathrm{x}}=0 \quad \Leftrightarrow \quad \frac{\partial h}{\partial x}=0$

where $\bar{q}_{\mathrm{o}}$ is the local volumetric oil production rate per meter well length. 
The model needs to handle two different regimes along the wellbore:

- $h(0, y, t)>z_{\mathrm{w}}-\mathrm{GOC}$ is locally above the wellbore, only oil (with dissolved gas) is produced. The local oil production rate can, at least in principle, be chosen arbitrarily large.

- $z_{\mathrm{w}}-d_{\mathrm{w}} \leq h(0, y, t) \leq z_{\mathrm{w}}-\mathrm{GOC}$ is in direct contact with the well at $x=0$ and free gas is produced locally in addition to oil. The oil production rate must now be limited to keep GOC above the bottom of the wellbore.

$h(0, y, t)=z_{\mathrm{w}}$ can be applied as an approximation for the boundary condition at $x=0$, as $d_{\mathrm{w}}$ is small compared to $z_{\mathrm{w}}$.

$z_{\mathrm{w}}$ is the vertical well location (top of the wellbore) and $d_{\mathrm{w}}$ is the diameter of the wellbore.

Two basic issues remain to complete the model:

- How to distribute the production rate along the wellbore

- How to find the gas and oil production rates where GOC is in contact with the well

In GORM, the distribution of the total production locally along the well is specified explicitly [4]. When there is no production of free gas, the local oil production can be written as:

$\bar{q}_{\mathrm{o}}(y, t)=u(t) K(y)$

where $K(y)$ is defined explicitly, and $u(t)$ can be regarded as a model input for total production.

For $K(y)$, a simple linear function is chosen:

$K(y)=(\beta-1) \frac{y}{L}+1$

where $\beta$ is a parameter to be adapted to the production data.

Where free gas is produced, some formulation of the gas production rate is required. The gas production must be limited. Another simple linear relation has been assumed: A reduction of the oil production is accompanied by a proportional increase in the production of free gas. The production rates can then be written as:

$\bar{q}_{\mathrm{o}}(y, t)=(1-\kappa) u(t) K(y)$

$\bar{q}_{\mathrm{g}}(y, t)=\gamma \kappa u(t) K(y)$

where $\bar{q}_{\mathrm{g}}$ is the local gas production rate, $\kappa$ is an oil reduction factor and $\gamma$ is a constant. The oil reduction factor is given by:

$\kappa(y, t)=\delta^{2}$ where the parameter $\delta$ is defined by:

$\delta=\left\{\begin{array}{cl}0 & , h(0, y, t)>z_{\mathrm{w}} \\ \frac{z_{\mathrm{w}}-h(0, y, t)}{d_{\mathrm{w}}}, & h(0, y, t) \leq z_{\mathrm{w}}\end{array}\right.$

By this definition, the oil production is reduced smoothly from "full" when GOC is above the well to zero if GOC should reach the bottom of the wellbore.

The central feature of the oil reduction factor is to keep $h(0, y, t)$ approximately at $z_{\mathrm{W}}$ when free gas is produced. Other possible formulations with this property would only have a minor influence on the computed results.

Now define the weighted (local) production rate by letting $\gamma \mathrm{m}^{2} / \mathrm{s}$ gas correspond to $1 \mathrm{~m}^{2} / \mathrm{s}$ oil production.

$\bar{q}_{\mathrm{a}}(y, t) \stackrel{\text { def }}{=} \frac{\bar{q}_{\mathrm{g}}(y, t)}{\gamma}+\bar{q}_{\mathrm{o}}(y, t)=u(t) K(y)$

The total weighted production is then given by:

$q_{\mathrm{a}}(t) \stackrel{\text { def }}{=} \int_{0}^{L} \bar{q}_{\mathrm{a}}(y, t) d y=u(t) \int_{0}^{L} K(y) d y=u(t) K_{\mathrm{I}}$

where

$K_{\mathrm{I}}$ (weighted) production integral, integral of $K(y)$ along the wellbore

The local oil and gas production rates can be given in terms of the weighted production rate:

$\frac{\bar{q}_{\mathrm{o}}}{q_{\mathrm{a}}}=\frac{(1-\kappa(y)) u(t) K(y)}{u(t) K_{\mathrm{I}}}=\frac{(1-\kappa(y)) K(y)}{K_{\mathrm{I}}}$

$\frac{\bar{q}_{\mathrm{g}}}{q_{\mathrm{a}}}=\frac{\gamma \kappa(y) u(t) K(y)}{u(t) K_{\mathrm{I}}}=\frac{\gamma \kappa(y) K(y)}{K_{\mathrm{I}}}$

and the corresponding production rates for the whole well can be written as:

$\frac{q_{\mathrm{o}}}{q_{\mathrm{a}}}=\frac{\int_{0}^{L}(1-\kappa(y, t)) K(y) d y}{K_{\mathrm{I}}}=\frac{K_{\mathrm{I}}-K_{\mathrm{red}}}{K_{\mathrm{I}}}$

$\frac{q_{\mathrm{g}}}{q_{\mathrm{a}}}=\frac{\int_{0}^{L} \gamma \kappa(y, t) K(y) d y}{K_{\mathrm{I}}}=\frac{\gamma K_{\text {red }}}{K_{\mathrm{I}}}$

where the oil reduction integral is defined by:

$K_{\text {red }}\left(q_{\mathrm{a}}, h\right)=\int_{0}^{L} \kappa(y, t) K(y) d y$

With the chosen formulation of the oil reduction factor, the integral will only depend on $h(0, y, t)$, i.e. the state 
of the reservoir at $x=0$. We will, however, assume that more general formulations might be chosen.

The total gas production in $\mathrm{Sm}^{3} / \mathrm{s}, Q_{\mathrm{g}}$, is the sum of the contribution from the free gas production and the contribution from gas dissolved in the reservoir oil.

$Q_{\mathrm{g}}=\frac{q_{\mathrm{g}}}{B_{\mathrm{g}}}+\frac{q_{\mathrm{o}}}{B_{\mathrm{o}}} R_{\mathrm{s}}=\frac{q_{\mathrm{a}}}{K_{\mathrm{I}}}\left(\frac{\gamma K_{\mathrm{red}}}{B_{\mathrm{g}}}+\frac{R_{\mathrm{s}}\left(K_{\mathrm{I}}-K_{\mathrm{red}}\right)}{B_{\mathrm{o}}}\right)$

where

$B_{\mathrm{g}} \quad$ Gas formation factor, $\mathrm{m}^{3} / \mathrm{Sm}^{3}$

One standard $\mathrm{m}^{3}$ gas has a volume of $B_{\mathrm{g}} \mathrm{m}^{3}$ in the reservoir

$B_{\mathrm{o}} \quad$ Oil formation factor, $\mathrm{m}^{3} / \mathrm{Sm}^{3}$

One standard $\mathrm{m}^{3}$ oil has a volume of $B_{\mathrm{o}} \mathrm{m}^{3}$ in the reservoir

$R_{\mathrm{S}} \quad$ solution of gas in the oil, $\mathrm{Sm}^{3} / \mathrm{Sm}^{3}$

One standard $\mathrm{m}^{3}$ oil from the reservoir contains $R_{\mathrm{S}} \mathrm{Sm}^{3}$ dissolved gas

The integral $K_{\mathrm{I}}$ is given by the function $K(y)$ and $K_{\text {red }}$ can be computed explicitly when the state of the reservoir is known. The weighted production rate is then given by Eq. 25 and the local oil production is given by Eq. 20. Hence, when the historical and/or planned gasproduction rate, $Q_{\mathrm{g}}$, is given, the boundary condition for Eq. 9 can be found and the partial differential equation (PDE) can be solved to predict the oil rate.

In GORM's program code, an equation similar to Eq. 25 is applied to solve for the reservoir oil production rate, $q_{\mathrm{o}}$ [4]. We have chosen here to derive an alternative formulation that will be applicable for more general choices for the oil reduction factor. Equation 25 also provides, for instance, the required equation for the more complex case where $\kappa$ depends on $q_{\mathrm{a}}$.

GORM uses a finite volume approach to discretize Eq. 9 to transform the PDE to a system of ordinary differential equations (ODEs) [4]. Actually, the current model version applies the 2D Dupuit-Forchheimer, Eq. 8. This modification did not in itself improve the predictions, in accordance with a recent model analysis [1]. It has, however, been kept as the increased computational time is minor. In addition, the simulated part of the reservoir can be extended in both ends in the $y$-direction along the wellbore, which is more realistic.

\subsection{Model analysis: 2D versus 1D flow}

towards the wellbore

A few years ago, it was decided to investigate the nearwell properties of the model. The 1D model in Eq. 9 should be compared to the corresponding $2 \mathrm{D}$ flow in the $x z$ plane, where the deviatoric pressure and the oil velocities are given by:

$k_{\mathrm{h}} \frac{\partial^{2} p}{\partial x^{2}}+k_{\mathrm{v}} \frac{\partial^{2} p}{\partial z^{2}}=0$

$v_{\mathrm{x}}=-\frac{k_{\mathrm{h}}}{\mu} \frac{\partial p}{\partial x}, \quad v_{\mathrm{z}}=-\frac{k_{\mathrm{v}}}{\mu} \frac{\partial p}{\partial z}$

The boundary conditions are specified pressure at GOC,

$p(x, h, t)=\Delta \rho g h(x, t)$

given oil velocity at the well, and no-flow across the remaining boundaries. A uniform distribution of the oil velocity was assumed at the well.

The equations were implemented in the finite element program COMSOL Multiphysics. The 2D pressure Eq. 26 was solved along with an arbitrary Lagrangian-Eulerian formulation to update the element grid to follow the time evolution of GOC.

A narrow oil reservoir with half width equal to $20 \mathrm{~m}$ and initial height $8 \mathrm{~m}$ was studied (surrounding rectangle in Fig. 2). The wellbore was located at $2 \mathrm{~m}$,

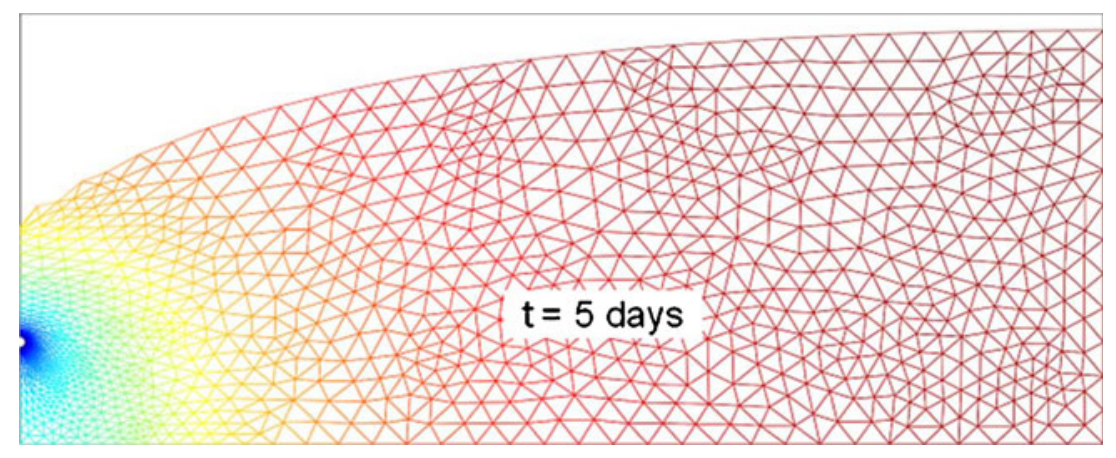

Fig. 2 Results from a numerical 2D simulation. The figure shows the deformed finite element grid. The colours illustrate the deviatoric pressure distribution ranging from $-16,400 \mathrm{~Pa}$ at the wellbore (dark blue) to 45,500 $\mathrm{Pa}$ in the upper right corner (brown). GOC is slightly unstable, i.e. some oscillations can be seen close to the wellbore 
and the well diameter was $20 \mathrm{~cm}$. The model first showed severe convergence problems. After the time integration parameters had been adjusted properly, the simulations showed smooth behaviour for some time. Then, the free boundary started to oscillate above the wellbore (Fig. 2). The oscillations grew and the solution procedure diverged after some more time steps.

The equations were then analysed for the isotropic case, and it was revealed that GOC would become unstable when the vertical oil velocity at GOC reached a critical value. The viscous fingering instability, c.f. for instance [2], was "rediscovered" for the special case with one viscous and one inviscid fluid. The simulations diverged as the model/code had not been adapted for such unstable cases.

The influence of the inviscid fluid is given by the boundary condition at GOC, Eq. 28, specifying the oil pressure. For isotropic conditions, the tangential velocity is then given by:

$v_{\mathrm{s}}=-c \frac{\partial p}{\partial s}=c g \Delta \rho \sin (\phi)=\frac{k}{\mu} g \Delta \rho \sin (\phi)$

where $v_{\mathrm{S}}$ is the tangential velocity at GOC in the direction towards the wellbore, $c=c_{\mathrm{h}}=c_{\mathrm{v}}$ is the isotropic diffusion coefficient, $k=k_{\mathrm{h}}=k_{\mathrm{v}}$ the corresponding permeability, and $\phi$ the inclination angle of GOC.

Maximum tangential velocity along GOC is achieved when the surface is vertical.

$\left(v_{\mathrm{s}}\right)_{\max }=\operatorname{cg} \Delta \rho$

A purely gravity-driven, porous oil flow cannot achieve a higher velocity. It is, however, possible to create higher velocities close to the well by supplying a sufficiently low well pressure. If the higher velocities extend to the surface, then the surface becomes unstable: If there is a small depression in the surface, there will be highest pressure gradient at the bottom of the dip. Hence, the bottom will try to move downwards faster than the remaining part of the interface. At low velocities, the dip will be filled by gravity flow, and the surface remains smooth. If the downwards velocity is higher than $\left(v_{\mathrm{s}}\right)_{\max }$, the gravity flow can not fill the depression, and a gas "finger" will rapidly stretch down to the well, Fig. 3. For the GOR model, such a dip can only be formed right above the well since the vertical oil velocity is highest there.

For the anisotropic case with different horizontal and vertical permeabilities, it can be shown that GOC will be unstable if the downwards velocity at GOC exceeds the critical value:

$v_{\mathrm{c}}=c_{\mathrm{v}} g \Delta \rho=\frac{k_{\mathrm{v}}}{\mu} g \Delta \rho$

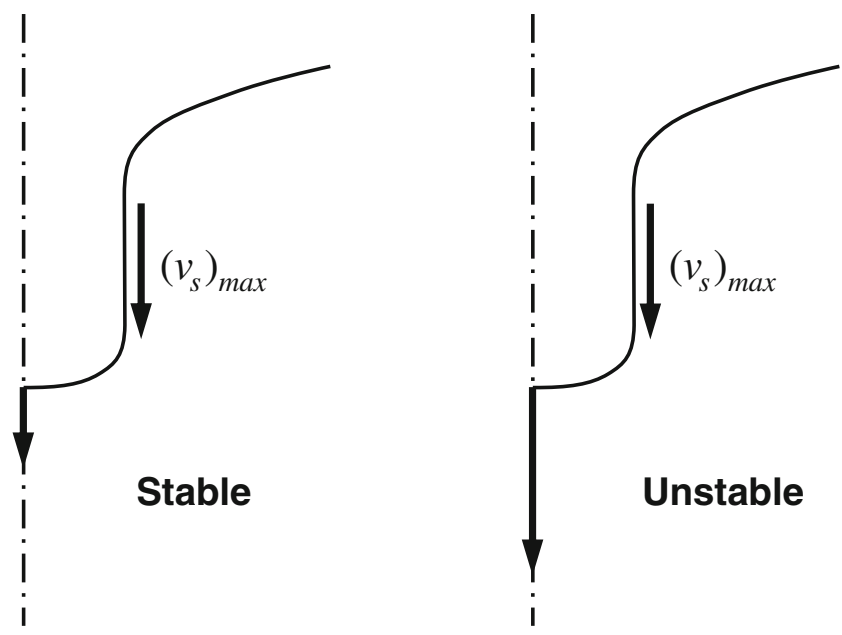

Fig. 3 A stable dip will be filled with oil, while an unstable one will grow deeper

The highest downwards velocity at GOC will be right above the wellbore. From the start, this velocity will be below the critical value, for normal production rates. Then, it will increase gradually as GOC is lowered. Unless the production rate is very low, GOC will be significantly above the wellbore when the critical rate is reached. Then, there will be gas breakthrough due to gas fingering, and free gas will be sucked into the well.

The production rate is limited by the total amount of gas production. Hence, after gas (fingering) breakthrough, the wellbore pressure will be reduced to control the production. The highest downwards oil velocity at GOC will be kept at the critical value. Higher weighted production will result in more free gas, while maximum oil velocity at GOC below the critical value would correspond to stable conditions with gas production below the target value.

From a modelling point of view, the oil production rate should be reduced to keep the maximum vertical velocity at GOC at the critical value, $v_{\mathrm{c}}$, after gas fingering breakthrough. This transition state should then be maintained until GOC reaches the wellbore. Then, the oil production should be further reduced to keep GOC approximately in this location at $x=0$.

For our test cases, the critical (superficial) velocity was $2 \mu \mathrm{m} / \mathrm{s}=1.2 \mathrm{~m} /$ week. The corresponding rate of change for GOC was $v_{\mathrm{c}} / \phi=v_{\mathrm{c}} / 0.2=10 \mu \mathrm{m} / \mathrm{s}=$ $0.86 \mathrm{~m} /$ day $=6.0 \mathrm{~m} /$ week.

Further analysis has been applied to show that the 2D model described by Eqs. 26 and 27 has generally four non-dimensional parameters:

- $\delta_{\mathrm{a}}=\sqrt{\frac{k_{\mathrm{h}}}{k_{\mathrm{v}}}} \frac{h_{0}}{W}$, aspect ratio, adjusted for the effect of different permeabilities 
- $\tilde{z}_{\mathrm{wc}}=\frac{z_{\mathrm{wc}}}{h_{0}}=\frac{z_{\mathrm{w}}-\frac{1}{2} d_{\mathrm{w}}}{h_{0}}$, non-dimensional vertical location of the well centre

- $\tilde{d}_{\mathrm{w} \_\mathrm{h}}=\frac{d_{\mathrm{w}}}{h_{0}}$, non-dimensional height of the well/nondimensional well diameter

- $\quad \tilde{d}_{\mathrm{w}_{-} \mathrm{W}}=\frac{d_{\mathrm{w}}}{W}$, non-dimensional width of the well

These four parameters determine the qualitative behaviour of the solution. The non-dimensional width of the well is not important and can be neglected. If the permeabilities are equal, the non-dimensional width can be expressed by the non-dimensional diameter and the aspect ratio. In this case it is no longer a free parameter.

The aspect ratio determines the relative strength of horizontal versus vertical flow and also determines the size of the near field. The non-dimensional vertical location of the well influences the flow field in the vicinity of the wellbore and hence the time evolution of GOC close to the well. The non-dimensional well diameter will basically influence the flow very close to the well, and can have a large impact on the pressure difference between the reservoir and the well. Such pressure difference is not an issue for the GOR model and this non-dimensional parameter is therefore not important.

Similar analysis for the one-dimensional model reveals that there are no non-dimensional parameters before gas breakthrough.

In addition to the non-dimensional parameters the quantities applied to scale the various variables are also (potential) free parameters. For the 2D equation, these parameters are:

- $h_{0}$, initial height of the oil reservoir

- $W$, half width of the oil reservoir

- $p_{0}=\Delta \rho g h_{0}$, typical pressure difference

- $\tau_{\mathrm{h}}=\frac{W^{2}}{\alpha h_{0}}=\frac{\mu \varphi}{\Delta \rho g} \frac{W^{2}}{k_{\mathrm{h}} h_{0}}$, time constant for horizontal flow, that is the time constant for draining the thin reservoir by gravitational flow.

As pressure values are not of interest for the GOR model, there are only three relevant free dimensional parameters. It can be shown that the $1 \mathrm{D}$ model has the same three free dimensional parameters. As the time constant for horizontal flow is the same for the 1D and the 2D models, the 1D approximation should be an appropriate approximation where this constant is relevant, i.e. for medium and long term simulations [1]. For very long-term simulations significant deviations from an initially rectangular reservoir shape are likely to be important and proper model predictions can not be expected.

The initial height of the reservoir is known from available information. GORM is then tuned to histori- cal production data by adjusting the parameters $W$ and $\alpha$. This is equivalent to adjusting $W$ and $\tau_{\mathrm{h}}$, as $\alpha$ can be expressed by the other free parameters and therefore can be applied instead of $\tau_{\mathrm{h}}$. In addition, the parameter $\beta$ is tuned. A fourth possible tuning parameter is $\gamma$, but this parameter is kept at a nominal value based on experience [4].

Two important non-dimensional parameters for the 2D model are not present in the current 1D approximation. These parameters can be combined with the dimensional ones to:

- $\tau_{\mathrm{v}}=\delta_{\mathrm{a}}^{2} \tau_{\mathrm{h}}=\frac{k_{\mathrm{h}}}{k_{\mathrm{v}}} \frac{h_{0}^{2}}{W^{2}} \frac{W^{2}}{\alpha h_{0}}=\frac{h_{0} k_{\mathrm{h}}}{\alpha k_{\mathrm{v}}}=\frac{\mu \phi}{\Delta \rho g} \frac{h_{0}}{k_{\mathrm{v}}}$, time constant for vertical flow, that is the time spent during a vertical "free fall" of oil from the top to the bottom of the initial reservoir height.

- $z_{\mathrm{wc}}=h_{0} \widetilde{z}_{\mathrm{wc}}$, vertical location of the well centre

As these parameters are important for the near-well flow, and hence for the short time behaviour, the current 1D model cannot be applied for accurate simulation of short-term variations.

\subsection{Model characteristics: 2D versus current 1D model}

Based on the model analysis, the current approximation does not take account of the following characteristics found in the 2D model:

- Effect of the aspect ratio, adjusted for possible different permeabilities

- Effect of the vertical location of the well centre, that is non-horizontal flow towards the well in the near-well region

- Ability to predict gas breakthrough due to viscous gas fingering

- Handling flow after (local) gas breakthrough before GOC reaches the wellbore

The ratio between the horizontal and the vertical permeabilities is a potential tuning parameter for short term time evolution, c.f. definition of $\delta_{\mathrm{a}}$ or $\tau_{\mathrm{v}}$ above.

\subsection{Flow regimes}

While the current model can handle two flow regimes locally along the wellbore, the analysis reveals that there will be three regimes:

- Stable oil flow with GOC above the wellbore - the maximum vertical oil velocity at GOC is below the stability limit.

- Transition flow, with gas fingering - the oil production rate tries to draw GOC downwards faster than 
the maximum gravity flow rate. Gas is sucked into the well due to viscous gas fingering and the oil flow is kept at the stability limit.

- Direct contact between the gas and the wellbore gas is sucked into the well. The oil production rate is limited by gravitational flow towards the wellbore.

Consider the early conditions for a reservoir and assume a suitable, constant gas production rate $\left(Q_{\mathrm{g}}=\right.$ constant). Further assume that the 2D model is applied for the flow towards the wellbore.

From the start, there will only be stable oil flow and gas coning will gradually develop. GOC will be lowest above the heel as the wellbore pressure is lowest here. After some time, the critical downwards velocity will be reached above the heel and there will be gas (fingering) breakthrough in this region. The oil production is then reduced as free gas is sucked into the well. Transition flow will start. First only right at the heel, but depending on how freely gas can be sucked, the transition flow region will gradually extend somewhat along the wellbore. Some model is required to describe how much free gas is produced locally when the oil production is reduced, for instance by assuming a linear relation, c.f. Eqs. 14 and 15.

Before GOC reaches the wellbore, the region with transition flow will gradually expand. Later, GOC will get in contact with the wellbore at the heel. The oil production must then be further reduced, and the production of free gas correspondingly increased. The higher gas production at the heel implies lower gas production further along the well and the flow state will change from transition flow to stable at the end of the transition region.

Gradually, GOC will get in contact with a larger and larger part of the wellbore, reducing the transition zone. The accompanying increase in the production of free gas implies that the transition zone is gradually reduced also from the other end, until it disappears. From now on, the zone where the gas is in direct contact with the wellbore will slowly expand towards the toe.

\section{Improved 1D approximation}

\subsection{Superposition of three flows}

In the following paragraphs, the modified model will be described for the case where the horizontal and vertical permeabilities are equal, i.e. $k_{\mathrm{h}}=k_{\mathrm{v}}=k$ and $c_{\mathrm{h}}=c_{\mathrm{v}}=c$. Flow in the $y$-direction will also be ignored and the flow towards the wellbore is then adequately described by:

$\nabla^{2} p=0$

$v_{\mathrm{x}}=-c \frac{\partial p}{\partial x}=-\frac{k}{\mu} \frac{\partial p}{\partial x}, \quad v_{\mathrm{z}}=-c \frac{\partial p}{\partial z}=-\frac{k}{\mu} \frac{\partial p}{\partial z}$

Oil flow in the reservoir is caused by two conditions: Lower pressure in the well and height variations of the GOC. Let the flow therefore be superimposed by the two terms:

- Flow due to oil production, i.e. flow towards the wellbore due to low pressure in the well. The boundary condition for the corresponding deviatoric pressure is $p=0$ at GOC.

- Flow due to gravity, i.e. flow caused by height variations of GOC, where the appropriate boundary condition at GOC is $p=\Delta \rho g h$.

The flow towards the wellbore is then approximated by radial flow towards a sink in the vicinity of the well. A mirror sink is added to get the correct boundary condition at the bottom of the reservoir. The flow due to the two wells will, however, violate the boundary conditions at GOC and at $x=W$. A third "sinkfix" flow is therefore added, to correct these boundary conditions.

Figure 4 shows how the total pressure is decomposed into two and then into three terms, together with the boundary conditions. The pressure equation and boundary conditions for the gravity flow are shown in Fig. 5, while Fig. 6 shows the equation satisfied by the flow caused by the two sinks and the two boundary

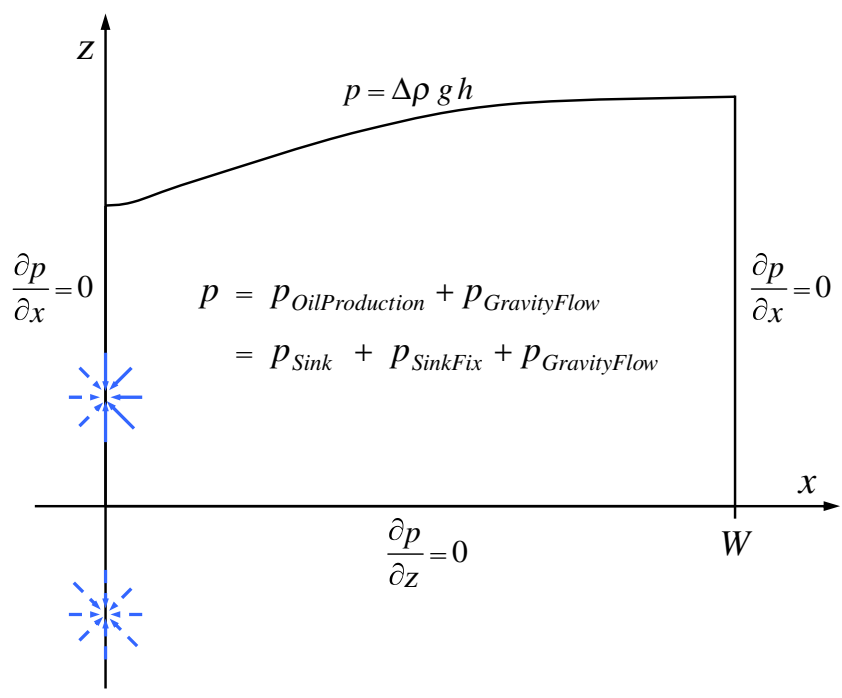

Fig. 4 Pressure as the sum of three terms 


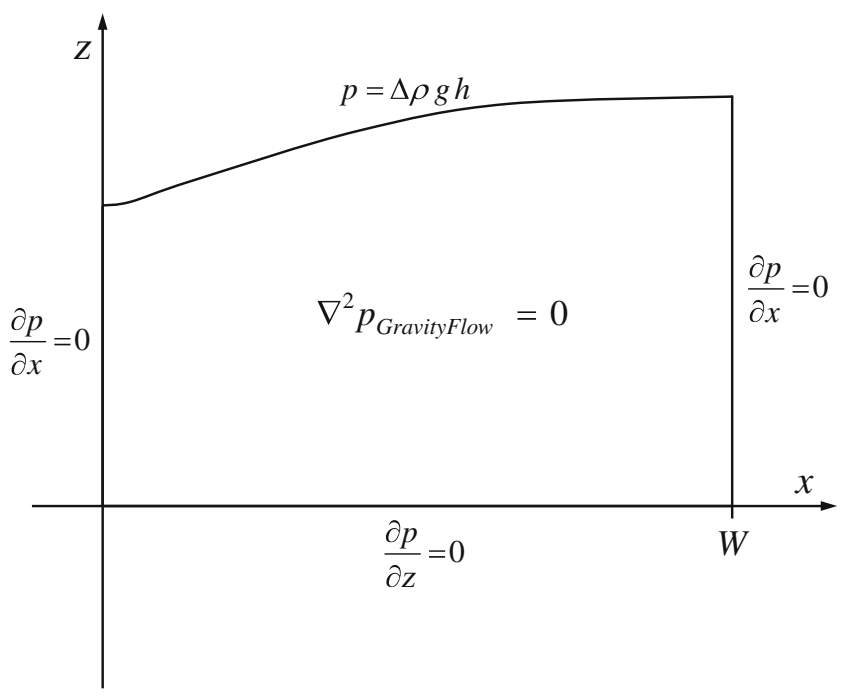

Fig. 5 Pressure due to gravity flow, no sinks

conditions satisfied by this flow. Finally, Fig. 7 shows the equation and the boundary conditions for the "sinkfix" flow.

\subsection{Horizontal flows}

The Dupuit assumption of vertical pressure equilibrium is a proper approximation for the gravity flow. Hence, the pressure is given by:

$p_{\text {gravity }}=\Delta \rho g h$

and the volume flow of oil per $\mathrm{m}$ well length in the $\mathrm{x}$-direction is given by:

$V_{\text {gravity_x }}=h v_{x}=-c \Delta \rho g h \frac{\partial h}{\partial x}$

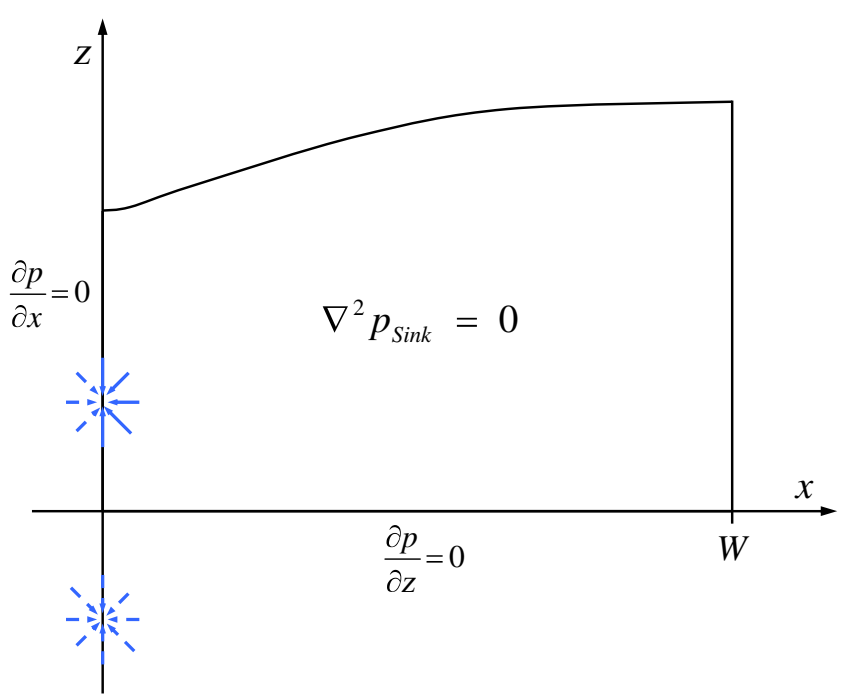

Fig. 6 Pressure from two sinks

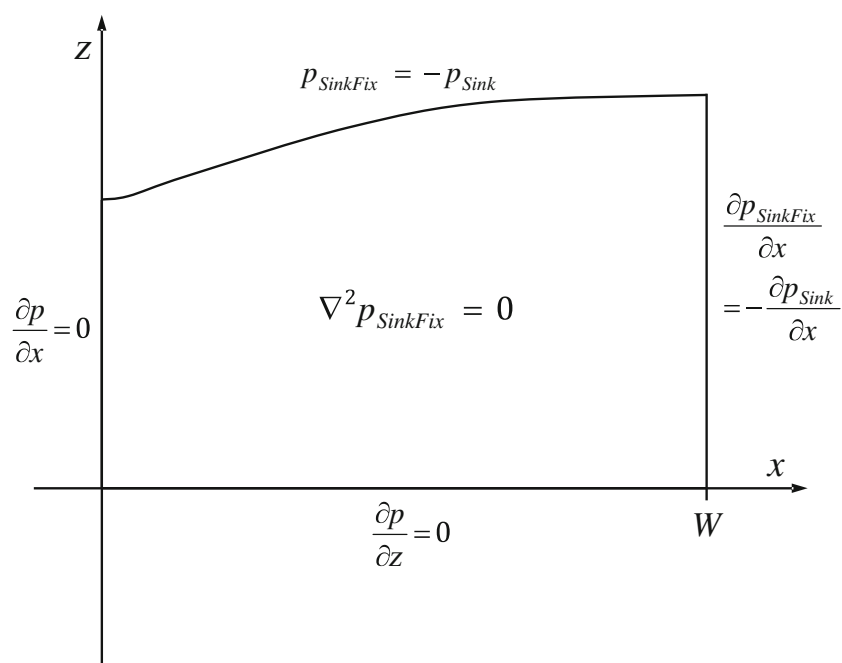

Fig. 7 Pressure to fix boundary conditions

The index gravity or GravityFlow is applied for the gravity flow.

The pressure for the flows towards the two 2D sinks can be written as:

$p_{\text {sink }}(x, z)=2 p_{*}+\frac{\bar{q}_{o}}{2 \pi c}\left(\ln \frac{r_{\mathrm{w}+}}{r_{*}}+\ln \frac{r_{\mathrm{w}-}}{r_{*}}\right)$

where $p_{*}$ is a reference pressure due to one of the sinks at a reference distance $r_{*}$ from this sink. For simplicity, let $p_{*}=0 \mathrm{~Pa}$ and $r_{*}=1 \mathrm{~m} . r_{\mathrm{w}+}$ and $r_{\mathrm{w}-}$ are the distances from the centre of the wellbore and the mirror wellbore, respectively:

$r_{\mathrm{w}+}=\sqrt{x^{2}+\left(z-z_{\mathrm{wc}}\right)^{2}}$

$r_{\mathrm{w}-}=\sqrt{x^{2}+\left(z+z_{\mathrm{wc}}\right)^{2}}$

Equation 36 has two terms. Each term describes the pressure due to pure radial flow towards the respective sink. The horizontal volume flow due to the wellbore sink is equal to the radial volume flow within the appropriate sector. Hence, the horizontal volume flow per $\mathrm{m}$ well length is given by:

$V_{\text {upper_sink_x }}=-\frac{\bar{q}_{\mathrm{o}}}{2 \pi}\left(\arctan \frac{h-z_{\mathrm{wc}}}{x}+\arctan \frac{z_{\mathrm{wc}}}{x}\right)$

Similarly, the horizontal volume flow (per $\mathrm{m}$ well length) due to the lower sink is:

$$
V_{\text {lower_sink_x }}=-\frac{\bar{q}_{\mathrm{o}}}{2 \pi}\left(\arctan \frac{h+z_{\mathrm{wc}}}{x}-\arctan \frac{z_{\mathrm{wc}}}{x}\right)
$$


The flow due to both sinks is then:

$$
\begin{aligned}
V_{\text {sink_x }} & =V_{\text {upper_sink_x }}+V_{\text {lower_sink_x }} \\
& =-\frac{\bar{q}_{o}}{2 \pi}\left(\arctan \frac{h-z_{\mathrm{wc}}}{x}+\arctan \frac{h+z_{\mathrm{wc}}}{x}\right)
\end{aligned}
$$

The Dupuit assumption will be used for the "sinkfix" flow. The pressure for this flow can then be written as:

$p_{\text {fix }}(x, t)=-p_{\text {sink }}(x, h(x, t), t)$

The horizontal flow is then given by:

$$
\begin{aligned}
V_{\text {fix } \_x} & =-h c \frac{\partial p_{\text {fix }}}{\partial x}=h c\left(\frac{\partial p_{\text {sink }}}{\partial x}+\frac{\partial p_{\text {sink }}}{\partial z} \frac{\partial h}{\partial x}\right) \\
& =\frac{\bar{q}_{\mathrm{o}} h}{2 \pi}\left(\frac{x+\left(h-z_{\mathrm{wc}}\right) \frac{\partial h}{\partial x}}{r_{\mathrm{w}+}^{2}}+\frac{x+\left(h+z_{\mathrm{wc}}\right) \frac{\partial h}{\partial x}}{r_{\mathrm{w}-}^{2}}\right)
\end{aligned}
$$

and the total horizontal oil flow per $\mathrm{m}$ well length is:

$V_{\mathrm{x}}=V_{\text {gravity_x }}+V_{\text {sink_x }}+V_{\text {fix_x }}$

Observe that when the Dupuit assumption is applied, the boundary conditions at $x=0$ and $x=W$ are not invoked. Due to symmetry of the pressure from the sinks, the condition at $x=0$ will be satisfied while the other condition can be somewhat violated. This discrepancy is easily handled by adjusting the boundary condition for the material balance.

\subsection{Material balance: equation for GOC}

The material balance is given by:

$\varphi \frac{\partial h}{\partial t}+\frac{\partial V_{\mathrm{x}}}{\partial x}=0$,

which can be written as:

$\frac{\partial h}{\partial t}=\alpha \frac{\partial}{\partial x}\left(h \frac{\partial h}{\partial x}\right)-\frac{1}{\varphi}\left(\frac{\partial V_{\text {sink_x }}}{\partial x}+\frac{\partial V_{\text {fix_x }}}{\partial x}\right)$

The boundary conditions are flow conditions:

$$
\begin{aligned}
V_{\mathrm{x}}(0) & =-\frac{1}{2} \bar{q}_{0} \\
V_{\mathrm{x}}(W) & =0
\end{aligned}
$$

If the boundary conditions are applied for $h$, then:

$$
\begin{aligned}
\frac{\partial h(0, t)}{\partial x} & =0 \\
V_{\text {gravity_x }}(W) & =\left[c \Delta \rho g h \frac{\partial h}{\partial x}\right]_{x=w} \\
& =-\left(V_{\text {sink_x }_{\_}}(W)+V_{\text {fix } \_ \text {X }_{1}}(W)\right)
\end{aligned}
$$

The flow condition at $x=0$ will be satisfied by the contribution from $V_{\text {sink_x }}$ while the contribution from $V_{\text {fix_x }}$ is zero.

It can be observed that the modified equation is equal to the original one, Eq. 9, plus two terms.

As for GORM, the PDE can be discretized by a finite-volume approach to derive an ODE-system to be solved by a standard ODE-solver. The complexity of the discretized modified model will be similar to the original one.

\subsection{Anisotropic conditions}

If the horizontal and vertical permeabilities differ, the appropriate $2 \mathrm{D}$ model is described by:

$k_{\mathrm{h}} \frac{\partial^{2} p}{\partial x^{2}}+k_{\mathrm{v}} \frac{\partial^{2} p}{\partial z^{2}}=0$

$v_{\mathrm{x}}=-c_{\mathrm{h}} \frac{\partial p}{\partial x} \quad, \quad v_{\mathrm{z}}=-c_{\mathrm{v}} \frac{\partial p}{\partial z}$,

instead of Eqs. 32 and 33.

The following coordinate transformation will change the problem to an isotropic one:

$\bar{z}=\lambda z=\sqrt{\frac{k_{\mathrm{h}}}{k_{\mathrm{v}}}} z$

In the transformed problem, all vertical parameters and the production rate have been changed by the same factor, $\lambda$, and the isotropic permeability is:

$\bar{k}=\sqrt{k_{\mathrm{h}} k_{\mathrm{v}}}$

The modified GOR model can then be applied on the transformed problem. Alternatively, the appropriate horizontal flows can be derived for the anisotropic case.

Hence, the modified model can also be applied for this type of anisotropic conditions.

\subsection{Analysis}

The appropriate non-dimensional version of Eq. 49 is:

$$
\left(\frac{k_{\mathrm{h}}}{k_{\mathrm{v}}} \frac{h_{0}^{2}}{W^{2}}\right) \frac{\partial^{2} \tilde{p}}{\partial \widetilde{x}^{2}}+\frac{\partial^{2} \tilde{p}}{\partial \widetilde{z}^{2}}=0
$$

Dupuit's assumption of vertical pressure equilibrium is valid when the factor $\left(\frac{k_{\mathrm{h}}}{k_{\mathrm{v}}} \frac{h_{0}^{2}}{W^{2}}\right)$ is small, c.f. for instance [1]. If the factor is less than 0.1, the second term in Eq. 53 will dominate. The argument can also be turned 
around: If the factor is greater than 0.1 , pressure variation in the vertical direction, and hence vertical flow, should not be ignored. The boundary for the near-well region can then be defined as the value of $W$ which makes the factor equal to 0.1 :

$W_{\mathrm{nw}}=h_{0} \sqrt{10 \frac{k_{\mathrm{h}}}{k_{\mathrm{v}}}}$

where $W_{\text {nw }}$ is the size of the near-well region. For isotropic conditions the half width of the near-well region is then some three times the reservoir height.

For the original GOR model, two important nondimensional parameters are missing: the location of the well centre (before gas breakthrough) and the aspect ratio (adjusted for possible different permeabilities).

For the modified model, the location of the well centre is evidently included. The aspect ratio will also be included as a non-dimensional parameter since the flow towards the two sinks is an exact solution to the original equation (but with different boundary conditions).

Our model modification will only affect the flow significantly in the near-well region. Outside this region the Dupuit assumption is valid and the "sinkfix" flow cancels the sink flow. The "sinkfix" flow, as given by Eq. 43 , is by definition exactly equal to minus the Dupuit-Forchheimer approximation for the sink flow.

Model simulations showed typical gas fingering instability for all cases that was run for sufficiently long time: First a smooth gas coning would be developed. Then the level of the first cell would suddenly move rapidly towards the wellbore, while the remaining part of GOC would develop as before. We are convinced that the modified 1D model will have a stability limit, similar to the critical downwards velocity for the 2D model, but a mathematical proof remains. Some preliminary, rather technical analysis showed, however, that the instability is likely to occur as GOC approaches the well. It remains to be studied how close the $1 \mathrm{D}$ instability will approximate the $2 \mathrm{D}$ one.

The modified model preserves the significant properties of the full 2D problem:

- The effect of 2D flow towards the wellbore.

- The near-well region, where 2D-flow should not be ignored, is the same as for the full 2D problem.

- An instability similar to the viscous gas fingering instability (not completely verified).

These properties indicate that the model will be a good approximation, but we can not preclude that further improvements can be found.

\subsection{Oil reduction factor}

The oil production rate must be reduced when GOC reaches the wellbore. As stated above, the main function is some controller that will keep GOC approximately at this location. For the new model GOC must be kept above the well centre. The formula in Eq. 16 can be kept:

$\kappa(y, t)=\delta^{2}$

provided the definition of $\delta$ is properly modified, for instance to:

$\delta=\left\{\begin{array}{cl}0, & h(0, y, t)>z_{\mathrm{ws}} \\ \frac{z_{\mathrm{ws}}-h(0, y, t)}{d_{\mathrm{ws}}}, & h(0, y, t) \leq z_{\mathrm{ws}}\end{array}\right.$

where $z_{\mathrm{ws}}$ is approximately equal to $z_{\mathrm{w}}$ (location for top of the wellbore) and $d_{\mathrm{ws}}$ is some constant such that

$d_{\mathrm{ws}}<z_{\mathrm{ws}}-z_{\mathrm{wc}}$

where $z_{\mathrm{wc}}$ is the vertical location of the well centre.

The oil production should also be reduced, and free gas produced, due to the gas fingering instability. It can be shown that the full problem becomes unstable when the vertical downwards superficial velocity exceeds the critical value given by, c.f. for instance [2]:

$v_{\mathrm{c}}=\frac{k_{\mathrm{v}}}{\mu} g \Delta \rho$

As an approximation in the discretized model we might limit the oil production when the rate of change for the level of the first cell grows too high, that is when

$\left|\dot{h}_{1}\right| \approx \frac{\nu_{\mathrm{c}}}{\phi}=\frac{k_{\mathrm{v}} g \Delta \rho}{\mu \phi}$

where $\dot{h}_{1}$ is the time derivative of the innermost cell. Such dependency on the time derivative implies that the oil reduction factor would depend on the local oil production rate. Hence, the oil reduction integral, $K_{\text {red }}$, would depend on the weighted production rate and the latter can not be computed explicitly by Eq. 25 .

Another approach is to detect viscous gas fingering by monitoring the difference between the oil level in the first and the second cell. The oil reduction factor can then be expressed as:

$\kappa(y, t)=\delta^{2}+\delta_{\mathrm{v}}^{2}$ 
where

$\delta_{\mathrm{v}}=\left\{\begin{array}{cl}0 & , h_{2}-h_{1}<\Delta h_{\mathrm{c}} \\ \frac{\left(h_{2}-h_{1}\right)-\Delta h_{\mathrm{c}}}{d_{\mathrm{c}}} & , h_{2}-h_{1} \geq \Delta h_{\mathrm{c}}\end{array}\right.$

where $\Delta h_{\mathrm{c}}$ defines the critical difference for onset of gas fingering in the model and $d_{\mathrm{c}}$ is another model parameter to be selected.

This choice for an additional term in the oil reduction factor will keep the difference $h_{2}-h_{1}$ between $\Delta h_{\mathrm{c}}$ and $\Delta h_{\mathrm{c}}+d_{\mathrm{c}}$ during viscous gas fingering. After some time, $h_{1}$ will be at the wellbore level. Then the other term in Eq. 60 will start to grow and reduction of oil production due to viscous fingering will gradually diminish.

Oil reduction due to gas fingering might also be controlled by the relative distance to the well centre, by defining:

$\delta_{\mathrm{v}}=\left\{\begin{array}{cl}0 & , \frac{h_{2}-z_{\mathrm{w}}}{h_{1}-z_{\mathrm{w}}}<\lambda_{\mathrm{c}} \\ \omega_{\mathrm{c}}\left(\frac{h_{2}-z_{\mathrm{w}}}{h_{1}-z_{\mathrm{w}}}-\lambda_{\mathrm{c}}\right) & , \frac{h_{2}-z_{\mathrm{w}}}{h_{1}-z_{\mathrm{w}}} \geq \lambda_{\mathrm{c}}\end{array}\right.$

where $\lambda_{\mathrm{c}}$ defines the critical ratio of the distances to the wellbore and $\omega_{\mathrm{c}}$ is a factor for tuning the model behaviour.

The last formulation for $\delta_{\mathrm{v}}$ may, after some time, keep $h_{1}$ suitably above the well centre. If this should be the case, the first term in Eq. 60 can be dropped.

The choice of formulation for the oil reduction factor is a control issue and not an essential modelling matter. The chosen formulation should:

- Detect viscous gas fingering

- Reduce local oil production during viscous gas fingering, while the level of the first cell approaches the well.

- Reduce local oil production to maintain GOC close to the well centre after GOC becomes sufficiently low.

The discretized model may, however, turn out to be sufficiently accurate without a controller for the viscous gas fingering instability. Our tests indicate that the resulting, discretized ODE system is numerically stable. It has only been observed that the level of the first cell suddenly moves rapidly towards the wellbore, without numerical problems. The time for this transition is comparatively small, provided the first computational cell (containing the well) is not too wide. The model could be further tested without a separate controller for the transition flow. It may turn out that it is sufficiently accurate for an appropriate choice of discretization close to the wellbore.

The transition flow will then only be implicitly included and the start and the end of this period will not be clearly defined. During the initial part of the transition there will be no production of free gas, when the level of the first cell moves rapidly towards the well. Then there will be gas production due to fingering for some time, until the levels of the neighbouring cells get close to the wellbore level.

Further model testing is required to determine what is a suitable strategy to handle the viscous gas fingering.

\subsection{Preliminary testing}

Figures 8, 9, 10, 11 show some results from preliminary model testing. The computational region for these simulations only covers the near-well region, i.e. a narrow reservoir. The tests were focused on the stable oil flow regime, before gas breakthrough. The parameters/data in Table 1 were applied. The parameters $\mu, k, \rho_{\mathrm{o}}, \rho_{\mathrm{g}}$, and $\varphi$ were applied to compute $\alpha$; while the parameters $L, B_{\mathrm{o}}, R_{\mathrm{S}}$ and $Q_{\mathrm{g}}$ were used to compute the local oil production rate, $\bar{q}_{\mathrm{o}}$, assuming zero production of free gas and uniform conditions along the wellbore.

A spatial discretization of $0.1 \mathrm{~m}$ was applied for both 1D models, while the 2D model applied an element grid somewhat coarser than shown in Fig. 2, but with smaller elements close to GOC above the well. The 1D models applied the routine ode15s in Matlab requiring a relative tolerance of $10^{-5}$ for the time stepping. The 2D model was run with a similar routine and with similar tolerance in COMSOL Multiphysics.

Figures 8 and 9 show results for a case with comparatively high production rate. The original GOR model clearly deviates from the 2D model, while the new 1D model is reasonably close. Some differences are found above the well.

Figure 9 shows that the level of the first cell moves quickly towards the wellbore after the ODE system has become unstable.

Figures 10 and 11 show the result for a case with lower production rate. For this case, there are also clear differences between GORM and the 2D simulation. The modified GOR model is very close to the $2 \mathrm{D}$ results except for $x<2 \mathrm{~m}$.

The tests are promising, but insufficient to show the performance of the improved model. Further work should include more comparative simulations before a complete, improved GOR model is tested against real production data. 
Fig. 8 Example 1: predicted shape of GOC after 2 days, $2 \mathrm{D}$ versus current and new 1D models

Fig. 9 Example 1: predicted shape of GOC after 5 days, $2 \mathrm{D}$ versus current and new 1D models

Fig. 10 Example 2: predicted shape of GOC after 10 days, $2 \mathrm{D}$ versus current and new 1D models

Fig. 11 Example 2: predicted shape of GOC after 16 days, $2 \mathrm{D}$ versus current and new 1D models
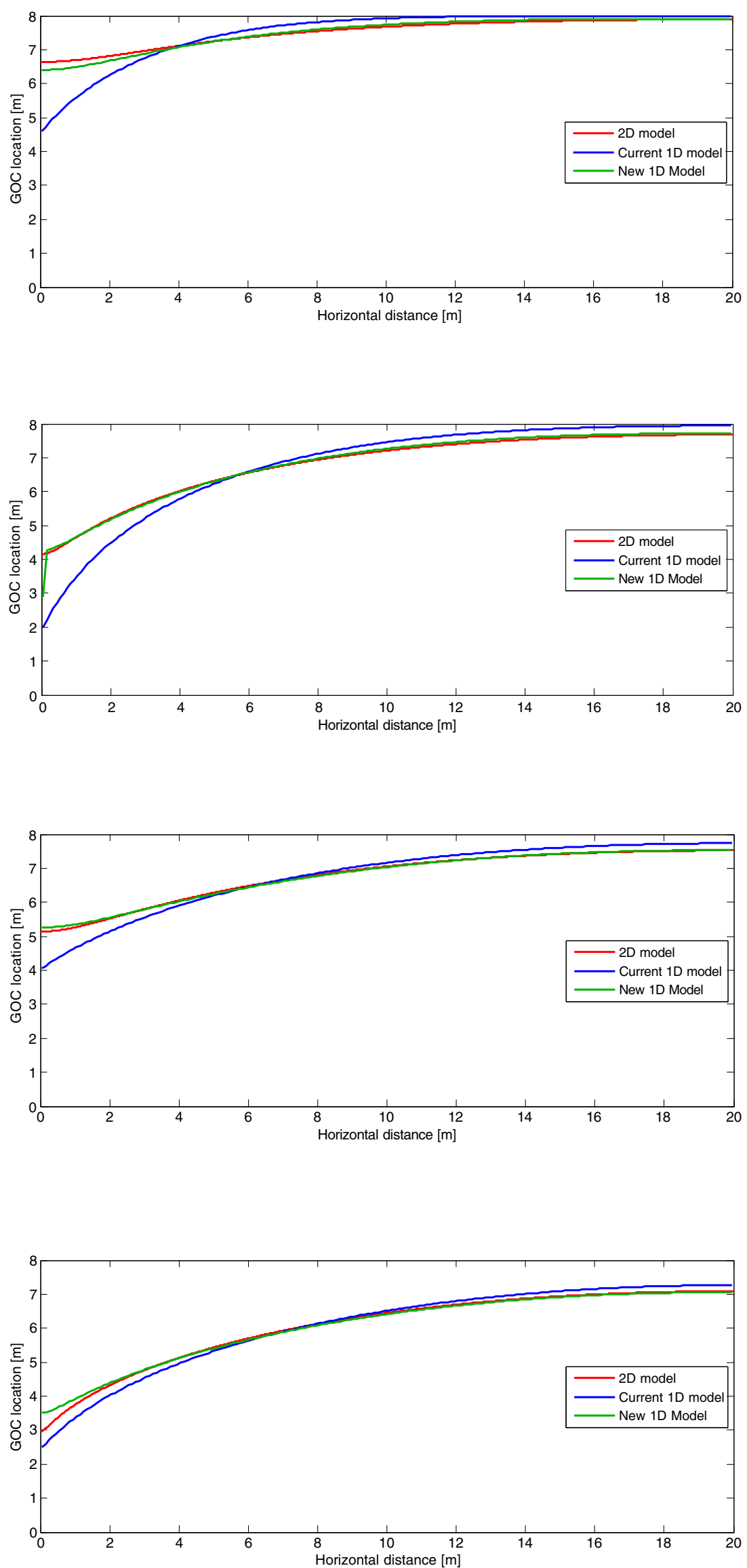
Table 1 Parameters/values for the simulations

\begin{tabular}{ll}
\hline Parameter & Value \\
\hline$W$, half width of the oil reservoir & $20 \mathrm{~m}$ \\
$h_{0}$, initial height of the oil layer & $8 \mathrm{~m}$ \\
$z_{\mathrm{W}}$, well location (top of wellbore) & $2 \mathrm{~m}$ \\
$d_{\mathrm{w}}$, diameter of wellbore & $20 \mathrm{~cm}$ \\
$\alpha$, parameter in the Dupuit-Forchheimer & $1.010^{-5} \mathrm{~m} / \mathrm{s}$ \\
$\quad$ equation & \\
$\mu$, oil viscosity (adapted to get & $0.0029607 \mathrm{~Pa} \mathrm{~s}$ \\
rounded value for $\alpha$ ) & $1.010^{-12} \mathrm{~m}^{2}$ \\
$k$, isotropic permeability & $800 \mathrm{~kg} / \mathrm{m}^{3}$ \\
$\rho_{\mathrm{o}}$, reservoir density of oil & $200 \mathrm{~kg} / \mathrm{m}^{3}$ \\
$\rho_{\mathrm{g}}$, reservoir density of gas & 0.2 \\
$\varphi$, effective porosity & $1000 \mathrm{~m}$ \\
$L$, length of the well & $1.17 \mathrm{~m}^{3} / \mathrm{Sm}^{3}$ \\
$B_{\mathrm{O}}$, oil formation factor & $60 \mathrm{Sm} / \mathrm{Sm}^{3}$ \\
$R_{\mathrm{s}}$, solution of gas in the oil & $100,000 \mathrm{Sm}^{3} / \mathrm{day}$ \\
$Q_{\mathrm{g}}$, total gas production, Example 1 & $50,000 \mathrm{Sm}^{3} / \mathrm{day}$ \\
$Q_{\mathrm{g}}$, total gas production, Example 2 & \\
\hline
\end{tabular}

\section{Discussion}

It should be kept in mind that GORM is not suitable for reservoir management. It has been developed for production optimization and focuses on the local behaviour for each horizontal well separately. The philosophy has been to keep the model simple, but sufficiently advanced to capture the essential reservoir behaviour associated with each well.

The simplifications start by assuming a simple geometry: symmetry and a rectangular shape for the initial state of the reservoir associated with the well. These assumptions are obviously wrong, but when model parameters are appropriately fitted to production data, the model can predict the basic time evolution for a limited period of time. The assumptions are definitely not adequate for very long simulations, but the model has performed remarkably well for medium term production optimization (weeks, months).

As the next simplification, only oil flow is computed within the reservoir. The model treats the gas as an inviscid fluid compared to the viscous oil. This assumption takes care of the flow within the oil reservoir, but some assumption for the gas production is needed at the well.

At any location along the wellbore only oil is produced before local gas breakthrough. The local oil production will be increased whenever the pressure in the well is lowered. After gas breakthrough the oil flow is limited, either by gravitational flow towards the well or the stability limit for viscous gas fingering. If the local well pressure is lowered, more free gas is produced while the oil production is preserved.
The simplest assumption for the gas production is a linear relation: Let the local production demand be. $\bar{q}_{\mathrm{a}}$. After gas breakthrough the oil production is lower than $\bar{q}_{\mathrm{a}}$, which can be expressed as:

$\bar{q}_{\mathrm{o}}=(1-\kappa) \bar{q}_{\mathrm{a}}$

The remaining production demand implies gas production, for which a linear relation gives:

$\bar{q}_{\mathrm{g}}=\gamma \kappa \bar{q}_{\mathrm{a}}$

The factor $\gamma$ is a constant to be determined. Since the gas is far less viscous than the oil, the value is obviously greater than one.

The oil reduction factor, $\kappa$, should in principle be determined by first computing the oil production for the appropriate boundary condition at $x=0$. For instance, when the gas is in direct contact with the wellbore, $h(0, y, t)=z_{\mathrm{w}}$ is a proper approximation for the boundary condition. The local oil production rate can then be computed and $\kappa$ can be determined by Eq. 63. But such a formulation implies that the equation structure depends upon the solution and the implementation will be comparatively complex. Formulating controllers to fulfil the boundary conditions approximately is definitely preferable from a computational point of view.

The pressure in the wellbore varies along the well with higher pressure in the toe than in the heel. Hence, the local production is highest at the heel. The simplest possible assumption is a linear function for the distribution of the production rate along the wellbore. A more complex description has been tested where a hydraulic model was included for the pipe pressure. The result was much longer computational time and no noticeable increase in simulation accuracy. The simple, linear function has therefore been kept.

For the oil flow within the reservoir, the Dupuit assumption of vertical pressure equilibrium has been made. This is a standard simplification for porous flow within thin regions, i.e. when the horizontal length scales are at least a few times greater than the height of the flow region.

Analysis shows that the Dupuit assumption is valid for the intermediate and far field regions, while it can be poor for the near field. It is specifically not appropriate to describe the flow close to a well. Hence, GORM can perform well for medium to long term simulations, while poor short term predictions are to be expected when changes in the near-well region are significant. The proposed, improved model addresses the near-well deficiencies of GORM, while keeping its medium/far field properties. 
For horizontal/vertical anisotropy, the ratio between the vertical and horizontal permeabilities is a model parameter. This parameter can be applied to adapt the model to historical short term variations. Such adaptation can be vital for proper short term behaviour. Hence, horizontal/vertical anisotropy should be included in the implementation.

The improved model is valid for flow perpendicular to the wellbore. This is a good approximation at some distance from the heel and the toe. To keep the model simple, the formulations should be applied along the complete length of the wellbore. If the model region is somewhat extended beyond the heel and toe, the appropriate equation will be:

$$
\begin{aligned}
\frac{\partial h}{\partial t}= & \alpha\left(\frac{\partial}{\partial x}\left(h \frac{\partial h}{\partial x}\right)+\frac{\partial}{\partial y}\left(h \frac{\partial h}{\partial y}\right)\right) \\
& -\frac{1}{\phi}\left(\frac{\partial V_{\text {sink_x }}}{\partial x}+\frac{\partial V_{\text {fix_x }}}{\partial x}\right)
\end{aligned}
$$

where anisotropic formulations should be implemented for the sink and the "sinkfix" terms.

\section{Conclusions}

Mathematical analysis explains why the current model to predict the gas/oil ratio, GORM, can perform remarkably well for medium term predictions (weeks, months), while short-term results (hours, days) can be poor.

An improved model is proposed that will take care of the current short term deficiencies. The new model

- Has the same medium and far field properties as the current model

- Improves the predictions of the gas oil contact (GOC) close to the wellbore
- Appears to be able to detect and handle gas breakthrough due to viscous gas fingering

- Includes an additional parameter that can be adapted to short term variations

- Only modifies the near-well properties of the current model

Based on our analysis, the new model should behave in the same way as the current one for medium and long term predictions, while short term variations should be significantly improved.

Preliminary simulations show improved predictions for the near-well region. Further simulations and testing are required to verify the capabilities of the new model.

Open Access This article is distributed under the terms of the Creative Commons Attribution Noncommercial License which permits any noncommercial use, distribution, and reproduction in any medium, provided the original author(s) and source are credited.

\section{References}

1. Halvorsen, S.A., Mjaavatten, A., Aasheim, R.: Analysis of an oil-rim reservoir model for prediction of the gas/oil production ratio. In: 6th IMA Conference on Modelling Permeable Rocks, Abstract Book, pp. 55-58 (2010)

2. Homsy, G.M.: Viscous fingering in porous media. Ann. Rev. Fluid. Mech. 29, 271-311 (1987)

3. Konieczek, J.: The concept of critical rate in gas coning and its use in production forecasting. SPEJ-Soc. Petrol. Eng. J. AIME. 20(722), 97-106 (1990)

4. Mjaavatten, A., Aasheim, R., Saelid, S., Gronning, O.: A model for gas coning and rate-dependent gas/oil ratio in an oil-rim reservoir. SPE Reserv Eval. Eng, Soc Petrol. Eng. 11, 842-847 (2008)

5. Muskat, M.: The flow of homogeneous fluids through porous media. McGraw-Hill, New York, London (1937) 